\title{
Identification of Substances by THz Spectroscopy and Multivariate Analysis
}

\author{
A. Pohl ${ }^{1,2}$, N. Deßmann ${ }^{1,2}$, K. Dutzi ${ }^{1}$, and H.-W. Hübers ${ }^{1,2}$ \\ ${ }^{1}$ Institut für Optik und atomare Physik, Technische Universität Berlin, 10623 Berlin, Germany \\ ${ }^{2}$ Intitue of Planetary Research, DLR e.V., Rutherfordstraße 2, 12489 Berlin, Germany
}

\begin{abstract}
The identification of different substances based on terahertz transmittance spectra and multivariate analysis (MVA) is demonstrated. Transmittance spectra are obtained by the use of a Fourier transform infrared spectrometer (FTIR). By means of principal component analysis (PCA) and partial least squares regression (PLS) the spectral data were analyzed in order to discriminate and to identify the substances from each other.
\end{abstract}

\section{INTRODUCTION}

$\mathrm{T}$ ERAHERTZ (THz) radiation shows much promise for security applications in terms of spectroscopic sensing and imaging. For example the detection of hidden threats or substances can prevent attacks and disasters at places where a large number of people congregate, such as airports. Various studies were made in this field of research over the last decade. This is mainly driven by the fact that many substances have characteristic absorption features in the $\mathrm{THz}$ range. The capabilities offered by $\mathrm{THz}$ radiation are explored for example by standoff detection of explosives [1], chemical sensing of drugs [1], [2], or imaging through packaging materials [3], [4].

We report on the measurement and analysis of transmittance spectra of various substances in the frequency range from 0.45 to $3 \mathrm{THz}$. A method for the classification of unknown substances based on MVA will be presented.

\section{RESULtS}

A variety of nine substances $\left(\mathrm{KCl}, \mathrm{NaCl}, \mathrm{NH}_{4} \mathrm{CO}_{3}, \mathrm{~K}_{2} \mathrm{CO}_{3}\right.$, sugar, fertilizer, acetylsalicylic acid, chocolate, fruit chew) as well as three mixtures of acetylsalicylic acid and sugar (mass ratios $1: 1,1: 3,3: 1$ ) were investigated by measuring their transmittance spectra with an FTIR. The samples were chosen in order to represent possible explosives as well as food products, which are commonly worn by persons. The substances were ground to fine powders, except chocolate and chew which were prepared as thin layers. Subsequently an amount of $0.1 \mathrm{gr}$ of the samples was placed between two HDPE windows $(30 \mathrm{~mm} \times 40 \mathrm{~mm}, 1 \mathrm{~mm}$ thickness $)$ in a dedicated sample holder. The transmittance spectra were obtained by an evacuated Bruker Vertex 80v FTIR. An external mercury-arc lamp was used as radiation source along with a broadband Mylar multilayer beam splitter of $6 \mu \mathrm{m}$ thickness. For detection a liquid-helium cooled silicon bolometer was used. Each substance was measured ten times. The measurement time was less than 100 seconds. For each of these measurements a new sample was prepared. A frequency range from 0.45 to $3 \mathrm{THz}\left(15\right.$ to $\left.100 \mathrm{~cm}^{-1}\right)$ was covered with a frequency resolution of one wavenumber $(\sim 30 \mathrm{GHz})$.

The spectra were analyzed by PCA in order to determine the relevant spectral characteristics and to differentiate between the substances. Within three PCA dimensions (called principal components, PCs) nearly $98 \%$ of the data variance can be explained (see Fig. 2). The relevant features for separation primarily belong to sugar and acetylsalicylic acid. Another feature which is important for the separation of the substances is the shape of the absorption spectra caused by scattering processes due to the sample grain.

For classification of unknown samples we build a PLS model based on eight spectra of each substance. The remaining two spectra were used as test samples for the PLS model prediction. PLS utilizes sample dependent information to create the model. We used the class membership in binary form $(0,1)$ for non-members and members for the pure substances, and ratios for the mixtures. The sample identification works well for the pure substances, which means that all tested samples could be assigned to the correct class. Even more ratios of pure substances within mixtures can be classified with good accuracy. This demonstrates the applicability of FTIR spectroscopy for the identification of various substances.

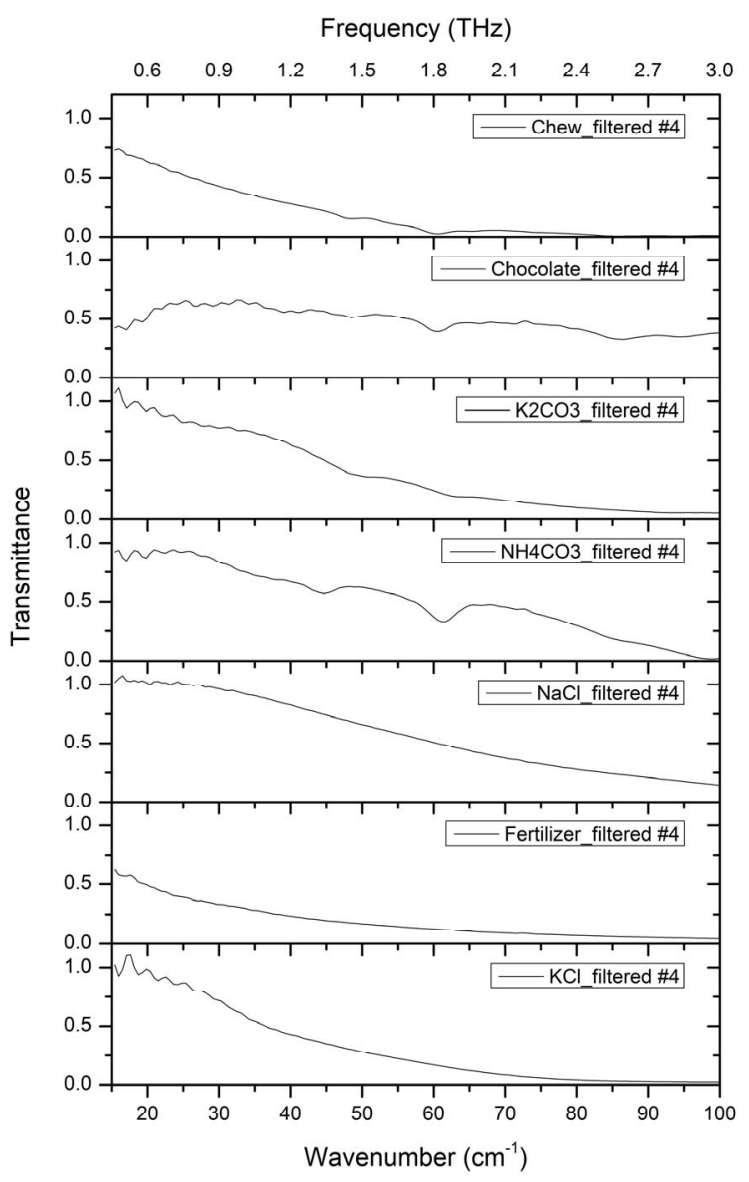

Fig. 1. Transmittance spectra of the samples (sugar, acetylsalicylic acid, and their mixtures are not shown). 

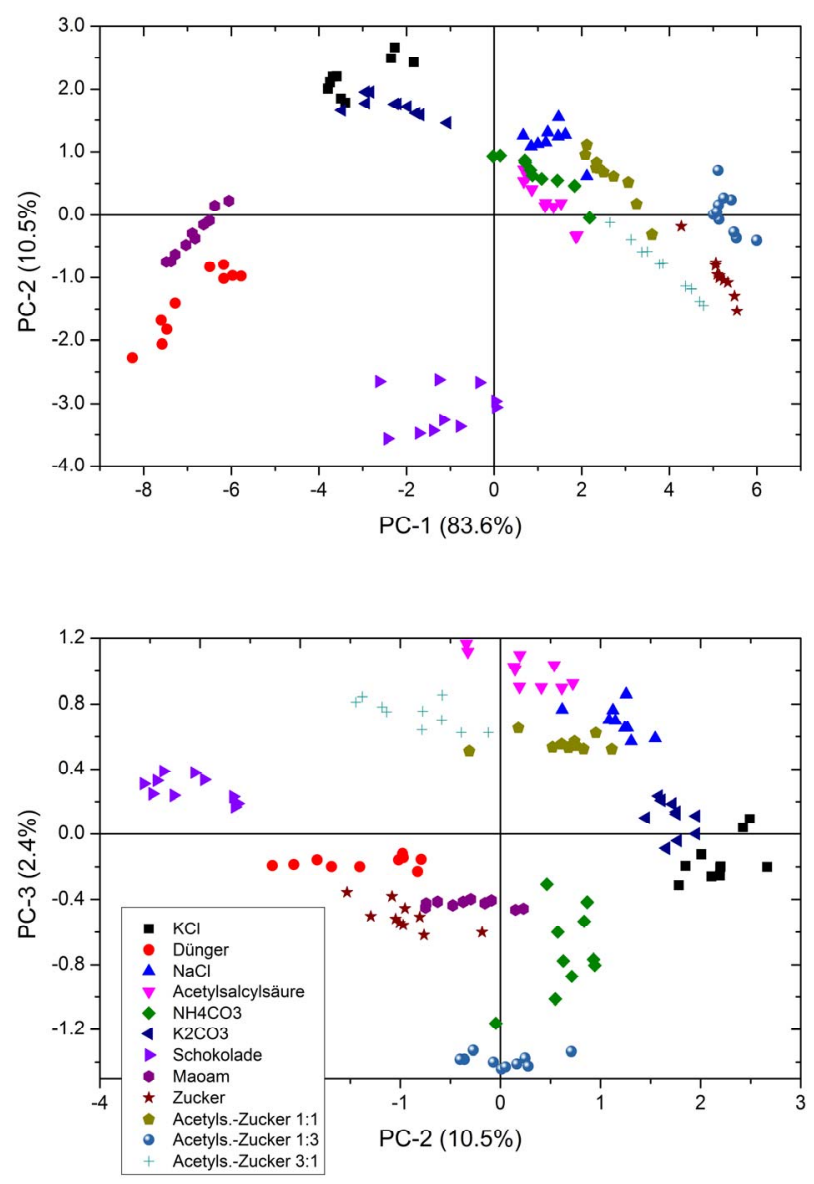

Fig. 2. Results of the PCA analysis of the raw transmittance data. It is worth to note that the data were not treated, i.e. no removal of standing waves or baseline artifacts was done. Shown are the scores of the first vs. second PC (top) and second vs. third PC (bottom). The data variance explained by the appropriate PC is given in brackets. Samples of the same substance class form clusters already when considering the first three PCs.

\section{SUMMARY}

We investigated a set of nine substances and three mixtures as a combination of two pure substances by utilizing FTIR spectroscopy. Two multivariate data analysis techniques were used to analyze the spectral data in the range of 0.45 to $3 \mathrm{THz}$. The substances can be distinguished from each other by applying the PCA. With PLS a model prediction was obtained where all substances can be assigned to the correct class. Also concentration ratios of pure substances within mixtures can be classified with good accuracy. The results demonstrate the applicability of FTIR spectroscopy for the identification of various substances.
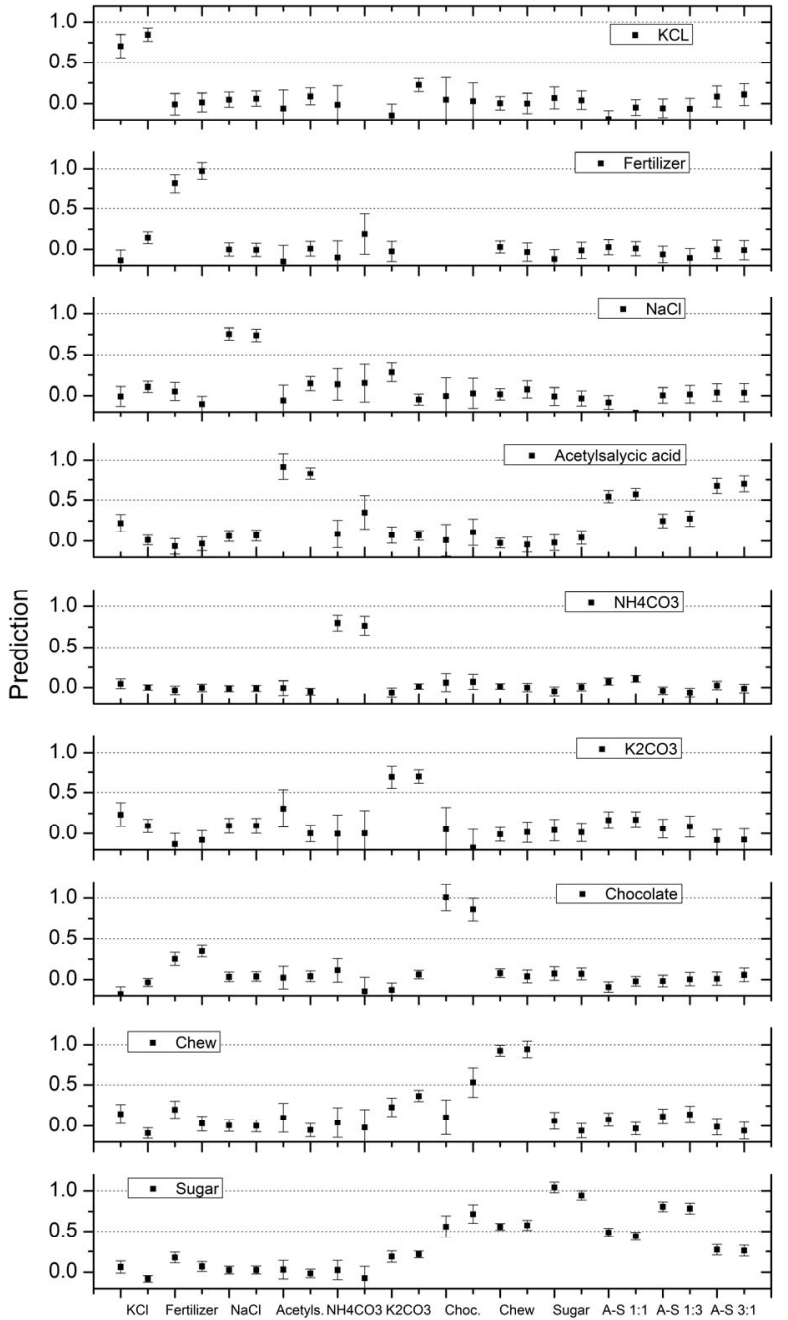

Substance

Fig. 3. PLS prediction of two samples for every respective substance. The prediction value defines the correlation probability for each sample. An ideal prediction would have a probability of 1 for the pure substance whereas predictions of samples not containing the substance would have a probability of 0 . Mixtures ideally give the ratio of the contained substance.

\section{REFERENCES}

[1] L. Ho, M. Pepper, and P. Taday Terahertz spectroscopy: signatures and fingerprints Nature Photonics, vol. 2, pp. 541 - 543, 2008

[2] J. F. Federici, B. Schulkin, F. Huang, D. Gary, R. Barat, F. Oliveira, and D. Zimdars, THz imaging and sensing for security applications - explosives, weapons and drugs, Semicond. Sci. Technol., vol. 20, no. 7, pp. S266-S280, June 2005

[3] N. Rothbart, H. Richter, M. Wienold, L. Schrottke, H. T. Grahn, and H.-W. Hübers, Fast 2-D and 3-D Terahertz imaging with a quantumcascasde laser and a scanning mirror, Terahertz Science And

Technology, vol. 3, no. 5, pp. 617-624, Sept. 2013

[4] K. Kawase, Y. Ogawa, and Y. Watanabe, Non-destructive terahertz imaging of illicit drugs using spectral fingerprints, Opt. Express, vol.11, no. 20, pp. 2549-2554, Oct. 2003. 